\title{
Intramolecular 1,3-dipolar cycloaddition reaction of novel 2-azetidinone-tethered alkenyl nitrile oxides
}

\author{
Benito Alcaide, ${ }^{* a}$ Pedro Almendros, ${ }^{b}$ and Elena Sáez ${ }^{a}$ \\ ${ }^{a}$ Departamento de Química Orgánica I, Facultad de Química, Universidad Complutense de \\ Madrid, 28040-Madrid, Spain. E-mail: alcaideb@quim.ucm.es \\ ${ }^{b}$ Instituto de Química Orgánica General, CSIC, Juan de la Cierva 3, 28006-Madrid, Spain. \\ E-mail: iqoa392@iqog.csic.es
}

Dedicated to Prof. Enrique Meléndez on the occasion of his $70^{\text {th }}$ birthday

(received 15 Sep 03; accepted 06 Apr 04; published on the web 07 Apr 04)

\begin{abstract}
Racemic as well as optically pure fused tricyclic $\beta$-lactams are regio and stereoselectively prepared via intramolecular nitrile oxide-alkene cycloaddition reaction of 2-azetidinone-tethered alkenyl oximes or nitro alkanes. The process is more efficient when the nitrile oxide moiety is separated by a methylenic group, rather than being directly linked to the $\mathrm{C}-4$ position of the fourmembered ring.
\end{abstract}

Keywords: Cycloaddition, $\beta$-lactam, nitrile oxide, tricycles

\section{Introduction}

A 2-azetidinone ( $\beta$-lactam) skeleton is well established as the key pharmacophore of $\beta$-lactam antibiotics, the most widely employed class of antibacterial agents. ${ }^{1}$ The importance and structural diversity of biologically active $\beta$-lactam antibiotics led to the development of many novel methods for the construction of appropriately substituted 2-azetidinones with attendant control of functional groups and stereochemistry. Tricyclic $\beta$-lactam antibiotics, generally referred to as trinems, are a new class of synthetic antibacterial agents featuring good resistance to $\beta$-lactamases and dehydropeptidases. ${ }^{2}$ Besides, the ever-growing new applications of 2azetidinones in fields ranging from enzyme inhibition ${ }^{3}$ to the use of these products as starting materials to develop new synthetic methodologies, ${ }^{4}$ has triggered a renewed interest in the building of new polycyclic $\beta$-lactam systems in an attempt to move away from the classical $\beta$ lactam antibiotic structures. ${ }^{5}$

The 1,3-dipolar cycloaddition between alkenes and dipoles is one of the most powerful tools for the synthesis of 5-membered heterocycles. ${ }^{6}$ Although many investigations have been made in 
this field into various types of systems, there is little information available regarding the regioand stereochemistry of reactions involving 2-azetidinone-tethered nitrile oxides as chiral building blocks on the 1,3-dipolar cycloaddition reaction, either inter or intramolecular. Thus, Sandanayaka has recently reported the intermolecular nitrile oxide cycloaddition of a 6(nitrileoxidomethyl) penam sulfone with alkenes and alkynes, ${ }^{7}$ while Hassner and Padwa described the intramolecular variant on $\beta$-lactams bearing the nitrile oxide tether at $\mathrm{N}-1 .{ }^{8}$ During the course of our ongoing project directed toward developing efficient routes to prepare chiral functionalized 2-azetidinones and their synthetic applications, ${ }^{9}$ we have used the inter and intramolecular nitrone-alkene(alkyne) cycloaddition of 2-azetidinone-tethered alkenyl(alkynyl)nitrones for the preparation of non-conventional $\beta$-lactams, ${ }^{10}$ as well as indolizidine and quinolizidine derivatives. ${ }^{11}$ In connection with this work, we wish to report here the intramolecular 1,3-dipolar cycloaddition reaction of 2-azetidinone-tethered alkenyl nitrile oxides.

\section{Results and Discussion}

Starting substrates, 4-oxoazetidine-2-carbaldehydes 1a-e, were prepared both in the racemic form and in optically pure form using standard methodology. Racemic compound $( \pm)$-1e was obtained as a single cis-diastereoisomer, following our one-pot method from $N, N$-di- $(p$ methoxyphenyl)glyoxal diimine. ${ }^{12}$ Enantiopure 2-azetidinones 2a-d were obtained as single cisenantiomers from the corresponding imine of $(R)-2,3-O$-isopropylideneglyceraldehyde, through a Staudinger reaction with the appropriate alkoxyacetyl chloride in the presence of $\mathrm{Et}_{3} \mathrm{~N}$. $^{13}$ Transesterification of 3-acetoxy-2-azetidinone (+)-2d with sodium methoxide in methanol gave alcohol $(+)-3$, which by treatment with allyl bromide under basic conditions gave the 2azetidinone (+)-4. Standard acetonide hydrolysis of compounds $\mathbf{2 a - c}$ and $(+)-\mathbf{4}$ followed by oxidative cleavage of the resulting diols smoothly provided 4-oxoazetidine-2-carbaldehydes 1ad (Figure 1). ${ }^{9}$ Having obtained the aldehydes, the next stage was set to carry out the oxime or nitro derivatives formation and the subsequent dipolar cycloaddition reactions.

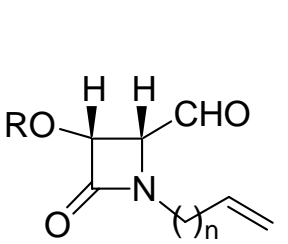

$(+)-1 \mathrm{a} n=1, \mathrm{R}=\mathrm{Ph}$

(+)-1b $n=2, R=P h$

$(+)-1 c n=2, R=M e$

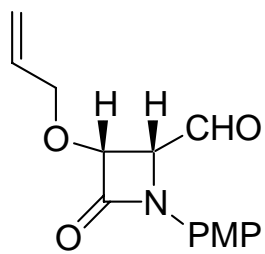

$(+)-1 d$

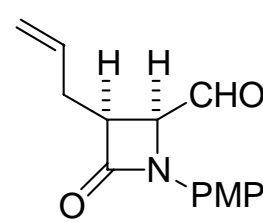

$( \pm)-1 e$

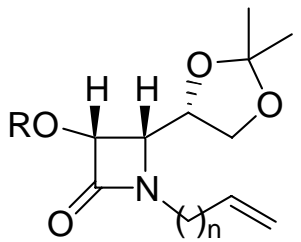

$(+)-2 a n=1, R=P h$

(+)-2b $\mathrm{n}=2, \mathrm{R}=\mathrm{Ph}$

$(+)-2 c n=2, R=M e$

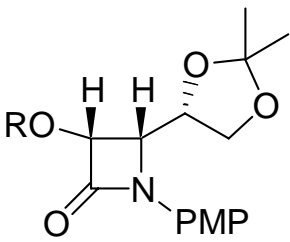

(+)-2d R = Ac

(+)-3 $\mathrm{R}=\mathrm{H}$

(+)-4 $\mathrm{R}=$ allyl

Figure 1 
The target molecules in the present study were derived from the intramolecular 1,3-dipolar cycloaddition of the appropriate $\beta$-lactam-tethered alkenyl nitrile oxide. This strategy should allow us to get a rapid access to unusual polycyclic $\beta$-lactams. Cycloadduct precursors, 2azetidinone-tethered oximes 5 , were smoothly prepared by the condensation of 4-oxoazetidine-2carbaldehydes 1 with hydroxylamine hydrochloride in benzene at room temperature in the presence of triethylamine (Scheme 1). Oximes 5 were obtained in almost quantitative yields as a mixture of isomers $(E / Z)$ and were used for next step without further purification. Importantly, the $\beta$-lactam ring stereochemistry was unaffected by this process.

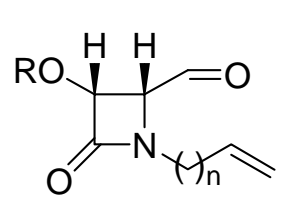

(+)-1a $\mathrm{n}=1, \mathrm{R}=\mathrm{Ph}$

$(+)-1 c \mathrm{n}=2, \mathrm{R}=\mathrm{Me}$

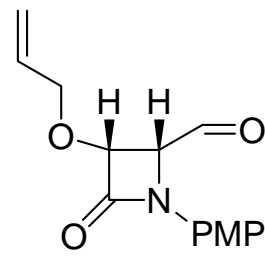

$(+)-1 d$

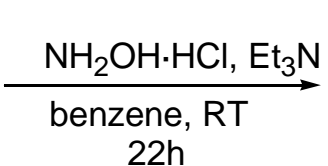

$22 \mathrm{~h}$<smiles>[R20]C1C(=O)N([R10]([H])=CC=C)[C@H]1C=NO</smiles>

(+)-5a (91\%) E/Z: 55/45

(+)-5b (100\%) E/Z: 60/40

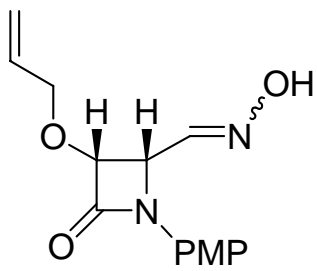

(+)-5c (100\%) E/Z: 55/45

\section{Scheme 1}

Nitroalkanes 8 were prepared following the procedure developed by Palomo for related substrates. $^{14}$ The reaction of nitromethane with 4-oxoazetidine-2-carbaldehyde $(+)-\mathbf{1 a}$ in the presence of triethylamine gave a diastereomeric mixture $(75: 25)$ of nitro-aldol product $(+)-6 a$ (Scheme 2).<smiles>C=CCN1C(=O)[C@H](Oc2ccccc2)[C@H]1C=O</smiles>

$(+)-1 a$

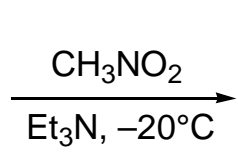

(+)-6a (92\%) sin/anti: 75/25<smiles>[2H][C@H]1C(=O)N(CC=C)[C@@H]1[C@H](O)C[N+](=O)[O-]</smiles>

\section{Scheme 2}


The dehydration of nitroaldols 6 was achieved when the hydroxy group was mesylated by methanosulfonyl chloride in the presence of triethylamine. There was no epimerization of the corresponding $\beta$-lactams under the reaction conditions as the ${ }^{1} \mathrm{H}$ NMR of the olefins 7 showed the presence of only a single $E$-isomer. The nitro olefins were reduced to nitro alkanes 8 by stirring with tributyltinhydride in dichloromethane/methanol (9:1) for 24 hours at room temperature (Table 1).

Table 1. Synthesis of nitroderivatives 7 and $\mathbf{8}$

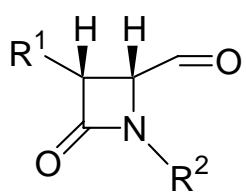

1

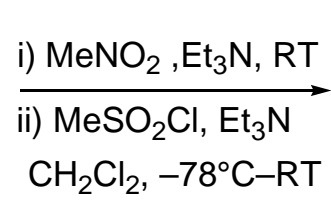

$\mathrm{CH}_{2} \mathrm{Cl}_{2},-78^{\circ} \mathrm{C}-\mathrm{RT}$

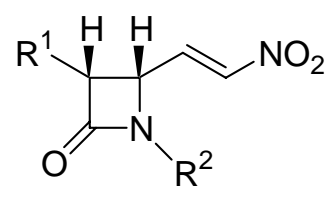

7

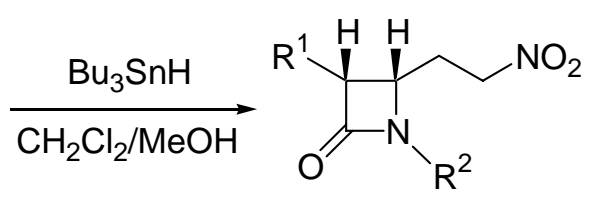

8

\begin{tabular}{ccccccc}
\hline Aldehyde & $\mathrm{R}^{1}$ & $\mathrm{R}^{2}$ & Nitro alkene & Yield (\%) & Nitro alkane & ${\text { Yield }(\%)^{a}}^{a}$ \\
\hline$(+)-1 \mathbf{a}$ & $\mathrm{OPh}$ & 2-propenyl & $(+)-7 \mathbf{a}$ & 70 & $(+)-8 \mathbf{a}$ & 70 \\
$(+)-\mathbf{1 b}$ & $\mathrm{OPh}$ & 3-butenyl & $(+)-7 \mathbf{b}$ & 60 & $(+)-8 \mathbf{b}$ & 60 \\
$( \pm)-\mathbf{1 e}$ & 2-propenyl & $\mathrm{PMP}^{b}$ & $( \pm)-7 \mathbf{c}$ & 72 & $( \pm)-8 \mathbf{c}$ & 81 \\
\hline
\end{tabular}

${ }^{a}$ Yield of pure, isolated product with correct analytical and spectral data. ${ }^{b} \mathrm{PMP}=4-\mathrm{MeOC}_{6} \mathrm{H}_{4}$.

Standard chlorination of oximes 5 with $N$-chorosuccinimide or sodium hipochlorite, followed by $\mathrm{Et}_{3} \mathrm{~N}$ treatment may generate nitrile oxides intermediates, which should afford the corresponding 1,3-dipolar cycloadducts. Disappointingly, poor yields of tricycles 9 were obtained under a variety of conditions (Table 2). 
Table 2. Intramolecular 1,3-dipolar cycloaddition reaction of 2-azetidinone-tethered alkenyl nitrile oxides

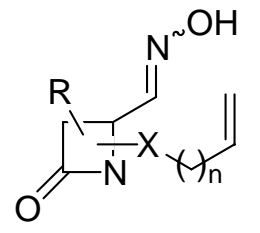

5

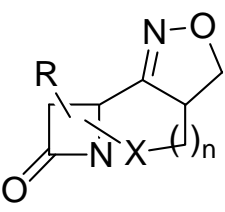

9

$$
\left(\mathrm{X}=\mathrm{O}, \mathrm{CH}_{2} ; \mathrm{n}=0,1\right)
$$

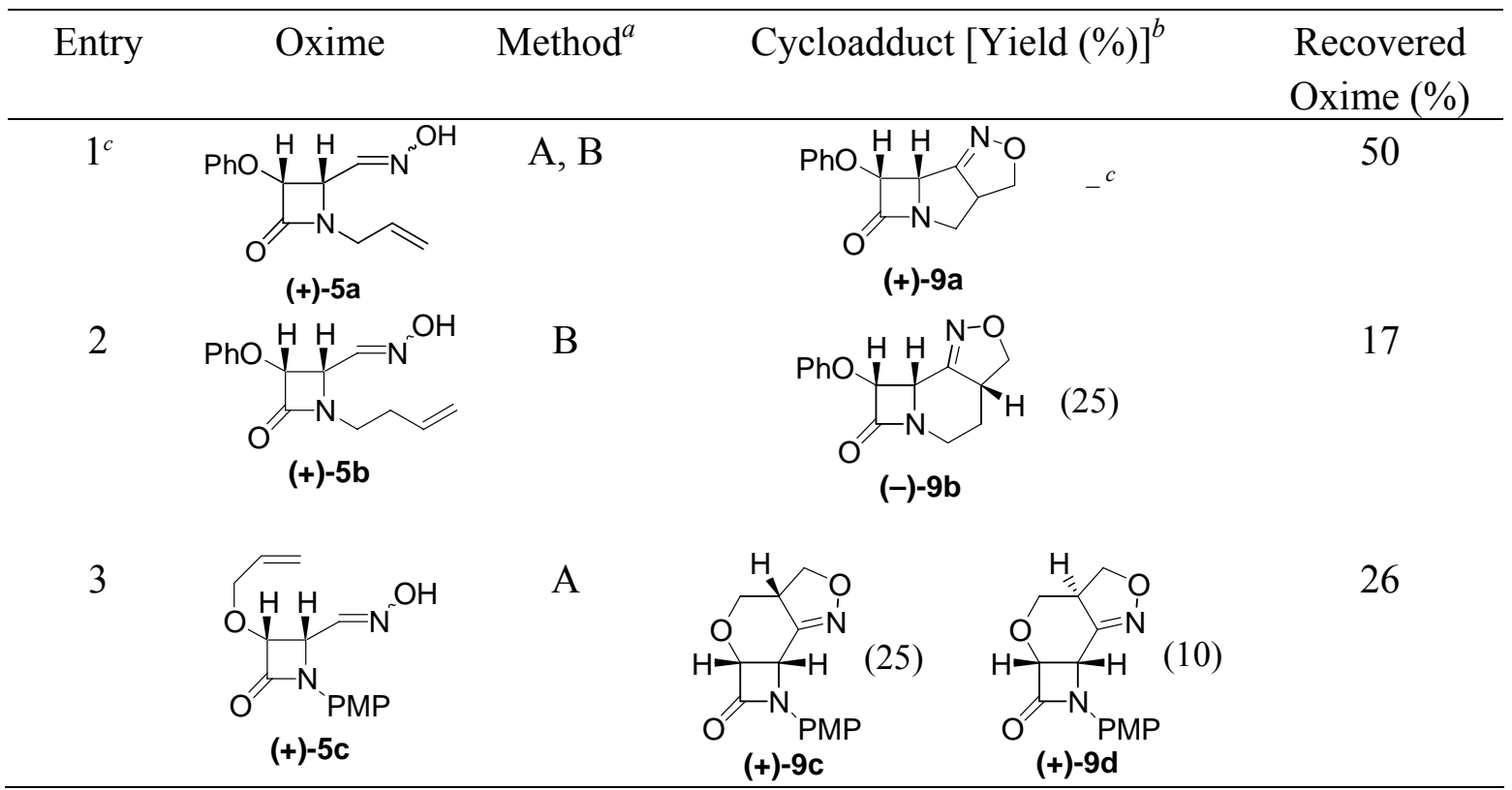

${ }^{a}$ Method A: NCS/Et 3 N, $\mathrm{CH}_{2} \mathrm{Cl}_{2}$, RT., 24h. Method B: $\mathrm{NaOCl} / \mathrm{Et}_{3} \mathrm{~N}, \mathrm{CH}_{2} \mathrm{Cl}_{2}, \mathrm{RT}$., 24h. ${ }^{b}$ Yield of pure, isolated product with correct analytical and spectral data. ${ }^{c}$ The corresponding cycloadduct was neither detected nor isolated.

The significant recovered amount of the starting oximes, point to difficulties in the nitrile oxide formation step. This fact could explain, at least in part, the low yields for cycloadducts 9. Results above show that the intramolecular 1,3-dipolar cycloaddition nitrile oxide reaction (INOC) of alkenyl 2-azetidinones, bearing nitrile oxide moieties directly linked to the C-4 position of the four-membered ring, is a modest entry to tricyclic 2-azetidinones with a sixmembered ring fused to the $\beta$-lactam nucleus, but it is not possible to obtain a central fivemembered ring.

Our next target was to use the nitroalkanes 8 as INOC reaction precursors to generate the corresponding nitrile oxides via Mukaiyama's method. ${ }^{15}$ Indeed, the treatment of nitro derivatives 8 with phenyl isocyanate under triethylamine catalysis, afforded the corresponding 
tricyclic isoxazolines 10 (Scheme 3 ). The reaction of nitro alkane $(+)-8 a$ proceeded in a total stereoselective fashion, achieving the tricyclic 2-azetidinone $(+)-\mathbf{1 0 a}$ in good yield $(83 \%)$ as a single isomer. The INOC reactions of the nitrile oxides derived from nitro compounds $(+)-\mathbf{8 b}$ and $( \pm)-\mathbf{8 c}$ were less stereoselective (Scheme 3). Diastereomers $( \pm)$-10c and $( \pm)$-10d were separated by column chromatography, while compound $\mathbf{1 0 b}$ was characterized as a mixture of unseparable isomers.

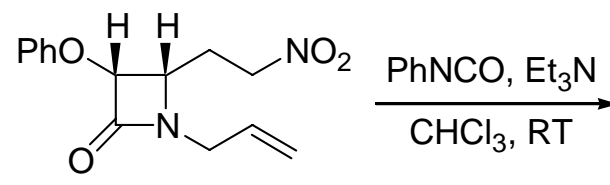

$(+)-8 a$<smiles></smiles>

(+)-10a (83\%)

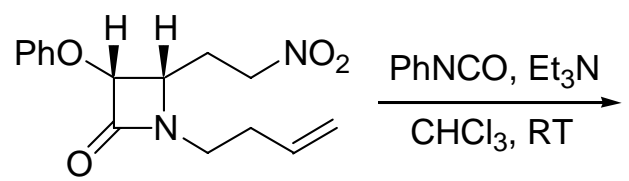

$(+)-8 b$<smiles>O=C1[C@H](O)[C@H]2CC3=NOC[C@@H]3CCN12</smiles>

10b (63:37, 50\%)

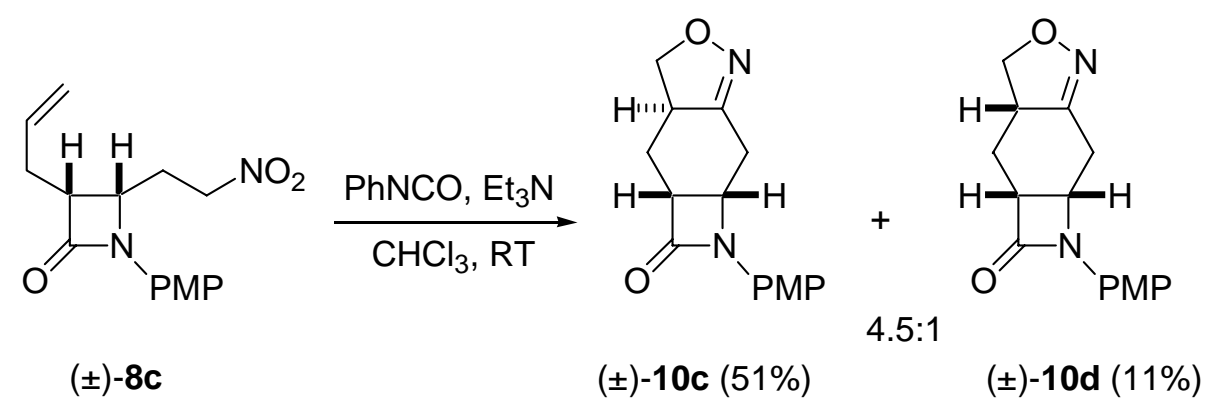

\section{Scheme 3}

The reaction of nitro aldol $\mathbf{6 a}$ deserves a special mention. On treating compound 6a with acetic anhydride and $\mathrm{Et}_{3} \mathrm{~N}$, tricycle 11 was obtained in $30 \%$ yield as a mixture (65:35) of diastereomers. The process must start through the generation of the dipole (nitrile oxide) with simultaneous protection of the hydroxyl group. Next, the 1,3-dipolar cycloaddition should take place followed by an elimination of acetic acid (Scheme 4). 


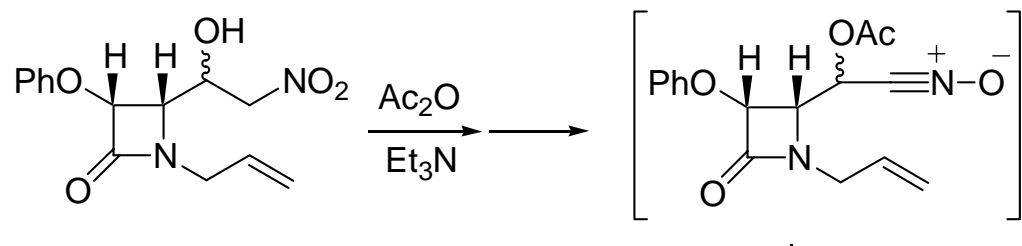

$6 \mathbf{a}$<smiles>O=C1[C@H](O)C2=CC3=NOC[C@@H]3CN12</smiles><smiles>CC(=O)O[C@H]1C2=NOC[C@H]2CN2C(=O)[C@H](C)[C@H]12</smiles>

$11(65 / 35,30 \%)$

\section{Scheme 4}

The nitrile oxide formation must be prior to the hydroxyl group elimination, because on trying to react the nitro alkene $(+)-7$ a under the above reaction conditions, or under other typical reaction conditions of dipole generation of diene (+)-7a was unaltered (Scheme 5).

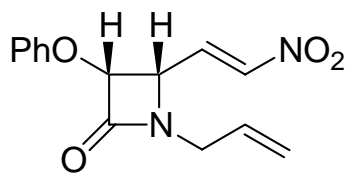

$(+)-7 a$
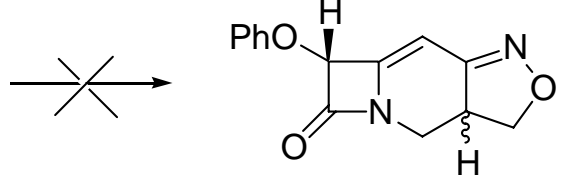

11

\section{Scheme 5}

The structure and stereochemistry of all compounds were assigned by NMR studies. The cisstereochemistry of the four-membered ring was set during the cyclization step to form the 2azetidinone ring, and it was transferred unaltered during the further synthetic steps. The tricyclic structures (by DEPT, HMQC, HMBC, and COSY) and the stereochemistry (by vicinal proton couplings and qualitative homonuclear NOE difference spectra) of $\beta$-lactams 9-11 were established by NMR one- and two-dimensional techniques. Thus, a syn-relative disposition between H4 and H9 was assigned for compound (-)-9b with a long distance coupling constant through four bonds $\mathrm{J}_{4,9}=1.5 \mathrm{~Hz}$. Furthermore, NOE-irradiation of $\mathrm{H} 4$ on compound (-)-9b resulted on enhancements on the signal corresponding to $\mathrm{H} 5$ eq $(3 \%), \mathrm{H} 2_{\mathrm{ax}}(2 \%), \mathrm{H} 3_{\mathrm{eq}}(2 \%)$, and $\mathrm{H} 9(1 \%)$, which is in agreement with the proposed stereochemistry. Irradiation of $\mathrm{H} 2$ on compound (+)-9c gave a NOE enhancement of $1 \%$ on $\mathrm{H} 7$, while the long distance coupling constant $\mathrm{J}_{2,7}=0.9 \mathrm{~Hz}$. Thus, a syn-relative disposition between $\mathrm{H} 2$ and $\mathrm{H} 7$ was assigned for tricycle (+)-9c (Figure 1). 


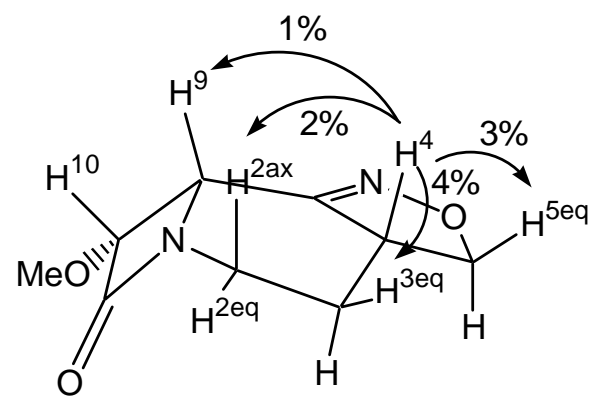

$(-)-9 b$

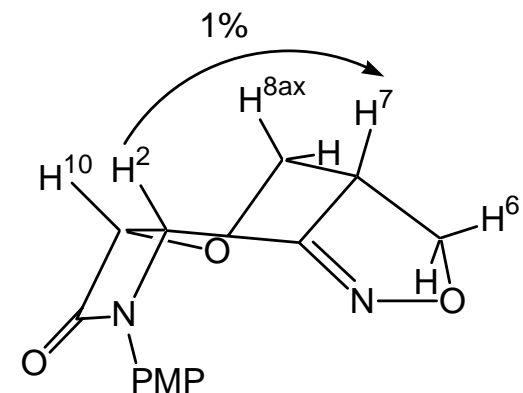

$(+)-9 c$

\section{Figure 1}

An anti-relative stereochemistry between the $\beta$-lactam hydrogens ( $\mathrm{H} 9$ and H10) and H3, as well as a trans-diaxial disposition between $\mathrm{H} 2_{\mathrm{ax}}$ and $\mathrm{H} 3$ was assigned for compound (+)-10a on the basis of coupling constants $\left(\mathrm{J}_{9,10}=4.1 \mathrm{~Hz}, \mathrm{~J}_{2 a x, 10}=1.1 \mathrm{~Hz}, \mathrm{~J}_{2 a x, 2 e q}=12.9 \mathrm{~Hz}, \mathrm{~J}_{2 a x, 3}=10.2 \mathrm{~Hz}\right)$. This stereochemistry was confirmed via NOE enhancement on $\mathrm{H} 8_{\text {eq }}(7 \%)$ and $\mathrm{H} 3(1.1 \%)$ on irradiating $\mathrm{H} 8_{\mathrm{ax}}$, and on $\mathrm{H} 2_{\mathrm{eq}}(12 \%), \mathrm{H} 4_{\mathrm{ax}}(3 \%)$, and $\mathrm{H} 9(1.3 \%)$ on irradiating $\mathrm{H} 2_{\mathrm{ax}}$. The stereochemistry for tricycles $( \pm)-\mathbf{1 0 c}$ and $( \pm$ )-10d was deduced in an analogous way (Figure 2$)$.

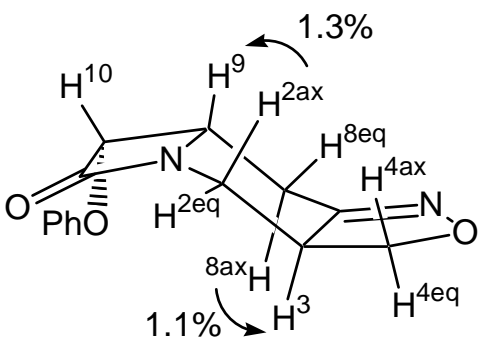

(+)-10a

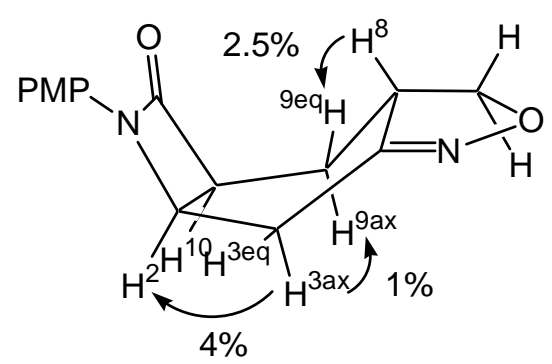

(士)-10c

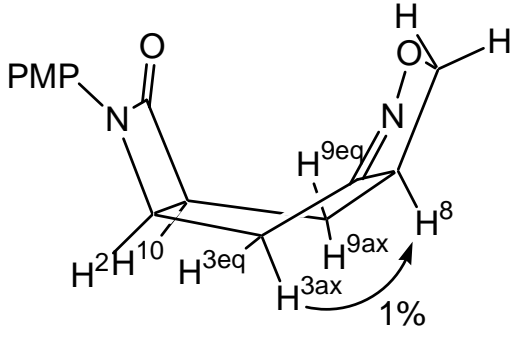

(土)-10d

Figure 2

\section{Conclusions}

The intramolecular 1,3-dipolar cycloaddition nitrile oxide reaction (INOC) of alkenyl 2azetidinones, bearing nitrile oxide moieties directly linked to the $\mathrm{C}-4$ position of the fourmembered ring, is a modest entry to tricyclic 2-azetidinones with a six-membered ring fused to the $\beta$-lactam nucleus. However, the INOC reaction of related alkenyl 2-azetidinones bearing nitrile oxide moieties separated by a methylenic group to the $\mathrm{C}-4$ position is a fair entry to this kind of $\beta$-lactams. 


\section{Experimental Section}

General Procedures. ${ }^{1} \mathrm{H}$ NMR and ${ }^{13} \mathrm{C}$ NMR spectra were recorded on a Bruker Avance-300, Varian VRX-300S or Bruker AC-200. NMR spectra were recorded in $\mathrm{CDCl}_{3}$ solutions, except otherwise stated. Chemical shifts are given in ppm relative to TMS $\left({ }^{1} \mathrm{H}, 0.0 \mathrm{ppm}\right)$, or $\mathrm{CDCl}_{3}\left({ }^{13} \mathrm{C}\right.$, $76.9 \mathrm{ppm}$ ). Low and high resolution mass spectra were taken on a HP5989A spectrometer using the chemical ionization modes (CI) unless otherwise stated. Specific rotation $[\alpha]_{\mathrm{D}}$ is given in deg per $\mathrm{dm}$ at $20{ }^{\circ} \mathrm{C}$, and the concentration $(c)$ is expressed in $\mathrm{g}$ per $100 \mathrm{~mL}$. All commercially available compounds were used without further purification.

\section{General procedure for the synthesis of 2-azetidinone-tethered oximes 5}

Hydroxylamine hydrochloride $(2.0 \mathrm{mmol})$ and triethylamine $(2.0 \mathrm{mmol})$ were sequentially added at room temperature to a well stirred solution of the corresponding 4-oxoazetidine-2carbaldehyde $(1.0 \mathrm{mmol})$ in benzene $(10 \mathrm{~mL})$. After the resulting suspension was stirred at room temperature overnight, the solvent was removed under reduced pressure. Then, the mixture was diluted with dichloromethane and washed with saturated aqueous $\mathrm{NaHCO}_{3}$ and water. The organic extract was dried $\left(\mathrm{MgSO}_{4}\right)$ and concentrated under reduced pressure to give analytically pure compounds 5 .

Oxime (+)-5b. From $229 \mathrm{mg}(1.41 \mathrm{mmol})$ of aldehyde (+)-1g, $247 \mathrm{mg}(100 \%)$ of compound 77b, as a $E / Z$ mixture $(60 / 40)$ of isomers, was obtained as a white solid. NMR data: $\delta_{\mathrm{H}}\left(\mathrm{CDCl}_{3}\right)$ 9.50 (brs, 1H, OH), $7.39(\mathrm{~d}, 0.6 \mathrm{H}, \mathrm{HC}=\mathrm{N}, J=8.1 \mathrm{~Hz}), 6.86(\mathrm{~d}, 0.4 \mathrm{H}, J=6.8 \mathrm{~Hz}, \mathrm{HC}=\mathrm{N}), 5.66$ $\left(\mathrm{m}, 1 \mathrm{H}, \mathrm{CH}=\mathrm{CH}_{2}\right), 5.07(\mathrm{~m}, 2 \mathrm{H}, \mathrm{CH}=\mathrm{CHH}), 4.97\left(\mathrm{dd}, 0.4 \mathrm{H}, \mathrm{H}-4,{ }^{3} J=4.6 \mathrm{~Hz}, J=6.8 \mathrm{~Hz}\right), 4.63$ (d, $\left.0.4 \mathrm{H}, \mathrm{H}-3,{ }^{3} \mathrm{~J}=4.6 \mathrm{~Hz}\right), 4.60$ (d, 0.6H, H-3, $\left.{ }^{3} \mathrm{~J}=4.5 \mathrm{~Hz}\right), 4.31$ (dd, $0.6 \mathrm{H}, \mathrm{H}-4,{ }^{3} \mathrm{~J}=8.1 \mathrm{~Hz},{ }^{3} \mathrm{~J}$ $=4.5 \mathrm{~Hz}), 3.43\left(\mathrm{~s}, 3 \mathrm{H}, \mathrm{O}-\mathrm{CH}_{3}\right), 3.39\left(\mathrm{~m}, 1 \mathrm{H}, \mathrm{N}-\mathrm{CH}_{2}\right), 3.15\left(\mathrm{~m}, 1 \mathrm{H}, \mathrm{N}-\mathrm{CH}_{2}\right), 2.27\left(\mathrm{~m}, 2 \mathrm{H}, \mathrm{CH}_{2}\right)$. $\delta_{\mathrm{C}}\left(\mathrm{CDCl}_{3}\right) 167.1(\mathrm{C}=\mathrm{O}, \mathrm{Z}), 166.9(\mathrm{C}=\mathrm{O}, M), 147.0(\mathrm{C}=\mathrm{N}, M), 146.8(\mathrm{C}=\mathrm{N}, m), 134.3$ $\left(\mathrm{HC}=\mathrm{CH}_{2}, M+m\right), 117.4\left(\mathrm{HC}=\mathrm{CH}_{2}, M+m\right), 84.7(\mathrm{C}-3, M+m), 58.9,58.7,57.6\left(\mathrm{O}-\mathrm{CH}_{2}, M+\right.$ $m)$, 51.6, $40.4\left(\mathrm{~N}-\mathrm{CH}_{2}, m\right), 39.9\left(\mathrm{~N}-\mathrm{CH}_{2}, M\right), 31.7\left(\mathrm{CH}_{2}, M\right), 31.6\left(\mathrm{CH}_{2}, m\right)$. IR $\left(\mathrm{KBr}, \mathrm{cm}^{-1}\right): v$ 3315,1751

Oxime (+)-5c. From $100 \mathrm{mg}(0.38 \mathrm{mmol})$ of aldehyde $(+)-3,106 \mathrm{mg}(100 \%)$ of compound 78, as a $E / Z$ mixture $(55 / 45)$ of isomers was obtained as a brown solid. NMR data: $\delta_{\mathrm{H}}\left(\mathrm{CDCl}_{3}\right) 8.75$ (brs, $1 \mathrm{H}, \mathrm{OH}), 7.54(\mathrm{~d}, 0.55 \mathrm{H}, J=8.1 \mathrm{~Hz}, \mathrm{HC}=\mathrm{N}), 7.36(\mathrm{~m}, 2 \mathrm{H}, \mathrm{Ar}), 6.97(\mathrm{~d}, 0.45 \mathrm{H}, J=7.3 \mathrm{~Hz}$, $\mathrm{HC}=\mathrm{N}), 6.86(\mathrm{~m}, 2 \mathrm{H}, \mathrm{Ar}), 5.91\left(\mathrm{~m}, 1 \mathrm{H}, 2 \mathrm{xCH}=\mathrm{CH}_{2}\right), 5.35\left(\mathrm{~m}, 5 \mathrm{H}, 4 \mathrm{xCH}=\mathrm{CH}_{2}, \mathrm{E}, \mathrm{Z}, \mathrm{H} 4 \mathrm{Z}\right), 4.96$ (d, $\left.0.45 \mathrm{H}, \mathrm{H}-3,{ }^{3} J=4.8 \mathrm{~Hz}\right), 4.92$ (d, 0.55H, H-3, $\left.{ }^{3} J=4.8 \mathrm{~Hz}\right), 4.76$ (dd, 0.55H, H-4, J= 8.1, 4.8 $\mathrm{Hz}), 4.23(\mathrm{~d}, 0.90 \mathrm{H}, J=18.7 \mathrm{~Hz}, \mathrm{OCHH}), 4.18(\mathrm{~d}, 1.10 \mathrm{H}, J=18.4 \mathrm{~Hz}, \mathrm{OCHH}), 3.78(\mathrm{~s}, 1.35 \mathrm{H}$, $\left.\mathrm{O}-\mathrm{CH}_{3}\right), 3.77\left(\mathrm{~s}, 1.65 \mathrm{H}, \mathrm{O}-\mathrm{CH}_{3}\right) \cdot \delta_{\mathrm{C}}\left(\mathrm{CDCl}_{3}\right) 163.4(\mathrm{NC}=\mathrm{O}, M), 163.3(\mathrm{NC}=\mathrm{O}, m), 156.7(\mathrm{C}=\mathrm{N}$, $m), 156.6(\mathrm{C}=\mathrm{N}, M), 147.7(\mathrm{C}), 133.1\left(\mathrm{HC}=\mathrm{CH}_{2}, M, m\right), 130.7(\mathrm{C}), 130.4(\mathrm{C}), 118.8\left(\mathrm{HC}=\mathrm{CH}_{2}\right.$, $m), 118.7\left(\mathrm{HC}=\mathrm{CH}_{2}, M\right), 118.5(\mathrm{CH}, M), 118.1(\mathrm{CH}, m), 114.5(\mathrm{CH}, m), 114.4(\mathrm{CH}, M), 82.3(\mathrm{C}-$ 3, $m), 82.2(\mathrm{C}-3, M), 72.4(\mathrm{C}-4, m), 72.3(\mathrm{C}-4, M), 56.9\left(\mathrm{O}-\mathrm{CH}_{2}, M+m\right), 55.5\left(\mathrm{O}-\mathrm{CH}_{3}, M+m\right)$. IR $\left(\mathrm{KBr}, \mathrm{cm}^{-1}\right): v$ 3284, 1733, 1514. 
Procedure for the synthesis of nitroaldol (+)-6a. To a solution of the aldehyde $(+)-1 a(150 \mathrm{mg}$, $0.65 \mathrm{mmol})$ in nitromethane $(1.5 \mathrm{~mL})$ cooled to $-20{ }^{\circ} \mathrm{C}$, was added triethylamine $(1.5 \mathrm{mmol})$. The resulting mixture was stirred at room temperature for $3 \mathrm{~h}$. The crude of reaction was poured into water. The organic layer was washed with $\mathrm{HCl} 5 \%, \mathrm{NaHCO}_{3}$ (satured solution) and brine, was dried $\left(\mathrm{MgSO}_{4}\right)$ and concentrated under reduced pressure. After chromatography of the residue eluting with hexanes/ethyl acetate (5:1), compound (+)-6a was obtained $(92 \%)$ as a mixture $(75 / 25)$ of isomers, as a colourless oil.

NMR data: $\delta_{\mathrm{H}}\left(\mathrm{CDCl}_{3}\right) 7.29(\mathrm{~m}, 4 \mathrm{H}, \mathrm{Ar}, M, m), 6.95(\mathrm{~m}, 6 \mathrm{H}, \mathrm{Ar}, M, m), 5.66(\mathrm{~m}, 2 \mathrm{H}$, $\left.H \mathrm{C}=\mathrm{CH}_{2}, M, m\right), 5.18(\mathrm{~m}, 6 \mathrm{H}, \mathrm{H} 3, \mathrm{HC}=\mathrm{CHH}, M, m), 4.50\left(\mathrm{~m}, 6 \mathrm{H}, \mathrm{H} 4, \mathrm{CH} H \mathrm{NO}_{2}, M, m\right), 3.85$ $\left(\mathrm{m}, 6 \mathrm{H}, \mathrm{NCHH}, \mathrm{CH} \mathrm{HNO}_{2}, M, m\right), 3.16$ (s broad, $\left.2 \mathrm{H}, \mathrm{OH}, M, m\right) . \delta_{\mathrm{C}}\left(\mathrm{CDCl}_{3}\right)$ 165.5, 156.9, 131.5, $130.8\left(\mathrm{HC}=\mathrm{CH}_{2}, m\right), 129.9(\mathrm{CH}, M, m), 123.4(\mathrm{CH}, m), 123.1(\mathrm{CH}, M), 120.0\left(\mathrm{HC}=\mathrm{CH}_{2}\right.$, $m), 119.5\left(\mathrm{HC}=\mathrm{CH}_{2}, M\right), 115.9(\mathrm{CH}, M, m), 81.2(\mathrm{C} 3, m), 80.1(\mathrm{C} 3, M), 77.6\left(\mathrm{CH}_{2} \mathrm{NO}_{2}, M, m\right)$, $69.0(\mathrm{CHOH}, M), 67.6(\mathrm{CHOH}, m), 58.5(\mathrm{C} 4, m), 58.0(\mathrm{C} 4, M), 44.6\left(\mathrm{NCH}_{2}, M\right), 44.2\left(\mathrm{NCH}_{2}\right.$, $m)$. IR $\left(\mathrm{CHCl}_{3}, \mathrm{~cm}^{-1}\right): v 3346,1745$.

\section{General procedure for the synthesis of nitroalkenes $\mathbf{7}$}

To a solution of the corresponding $\beta$-lactam $(1.0 \mathrm{mmol})$ in nitrometane $(1.5 \mathrm{~mL})$ was added triethylamine $(1.5 \mathrm{mmol})$, and the resulting mixture was stirred at room temperature until completion (typically $3 \mathrm{~h}$ ). Evaporation of the solvent under reduced pressure gave a residue, which was dissolved in dried dichloromethane $(4.5 \mathrm{~mL})$ and dropwise added at $-78{ }^{\circ} \mathrm{C}$ to a mixture of triethylamine $(0.41 \mathrm{~mL}, 3.0 \mathrm{mmol})$ and methanesulfonyl chloride $(3.0 \mathrm{mmol})$. After stirring for $30 \mathrm{~min}$ at $-78{ }^{\circ} \mathrm{C}$, triethylamine $(3.0 \mathrm{mmol})$ was added to the solution at $-50{ }^{\circ} \mathrm{C}$. The resulting mixture was gradually warmed up to room temperature during $7 \mathrm{~h}$, poured into water, and extracted with dichloromethane. The organic layer was washed with $0.1 \mathrm{~N} \mathrm{HCl}(3 \times 4.0 \mathrm{~mL})$ and then with aqueous $\mathrm{NaHCO}_{3}(4.0 \mathrm{~mL}$, saturated solution). The organic layer was separated and dried $\left(\mathrm{MgSO}_{4}\right)$. Evaporation of the solvent at reduced pressure gave the nitroalkenes 7, which were purified by chromatography on silica gel.

Nitroalkene (+)-7a. From $266 \mathrm{mg}(1.15 \mathrm{mmol})$ of aldehyde (+)-1a, $220 \mathrm{mg}(70 \%)$ of compound $(+)-7 a$ was obtained as a yellow solid after purification by flash chromatography (hexanes/ethyl ethyl acetate 1:1). Mp: $99-100{ }^{\circ} \mathrm{C}$ (hexanes/ethyl acetate). $[\alpha]_{\mathrm{D}}=+9.7\left(c 0.6, \mathrm{CHCl}_{3}\right) . \mathrm{NMR}$ data: $\delta_{\mathrm{H}}\left(\mathrm{CDCl}_{3}\right) 7.26(\mathrm{~m}, 7 \mathrm{H}, \mathrm{Ar}$ and $\mathrm{CHH}=\mathrm{CHNO}), 5.70\left(\mathrm{~m}, H \mathrm{C}=\mathrm{CH}_{2}\right), 5.46\left(\mathrm{~d}, \mathrm{H}-3,{ }^{3} J=4.6\right.$ $\mathrm{Hz}), 5.29(\mathrm{~m}, 2 \mathrm{H}, \mathrm{HC}=\mathrm{CHH}), 4.60\left(\mathrm{ddd}, \mathrm{H}-4,{ }^{3} J=7.1 \mathrm{~Hz},{ }^{3} J=4.6 \mathrm{~Hz},{ }^{3} J=0.5 \mathrm{~Hz}\right), 4.13\left(\mathrm{ddt},{ }^{2} J\right.$ $=15.4 \mathrm{~Hz}, J=5.6 \mathrm{~Hz}, J=1.3 \mathrm{~Hz}, \mathrm{~N}-\mathrm{CH} H), 3.71\left(\mathrm{ddt},{ }^{2} J=15.4 \mathrm{~Hz}, J=6.9 \mathrm{~Hz}, J=1.1 \mathrm{~Hz}\right.$, $\mathrm{NCHH}) . \quad \delta_{\mathrm{C}}\left(\mathrm{CDCl}_{3}\right) 164.3(\mathrm{NC}=\mathrm{O}), 156.6(\mathrm{C}), 142.9\left(\mathrm{CH}=\mathrm{CHNO}_{2}\right), 135.0\left(\mathrm{CH}=\mathrm{CHNO}_{2}\right)$, $130.3\left(\mathrm{CH}=\mathrm{CH}_{2}\right), 129.7(\mathrm{CH}), 122.9(\mathrm{CH}), 120.3\left(\mathrm{CH}=\mathrm{CH}_{2}\right), 115.4(\mathrm{CH}), 82.3(\mathrm{C}-3), 55.3(\mathrm{C}-4)$, $43.7\left(\mathrm{~N}-\mathrm{CH}_{2}\right)$. IR (KBr, cm-1): $v$ 1751, 1647. MS (EI), m/z : $274\left(\mathrm{M}^{+}, 89\right), 228$ (92), 181 (50).

Nitroalkene (+)-7b. From $210 \mathrm{mg}(0.86 \mathrm{mmol})$ of aldehyde $(+)-1 \mathbf{b}, 169 \mathrm{mg}(60 \%)$ of compound $(+)-7 \mathbf{b}$ was obtained as an orange oil after purification by flash chromatography (hexanes/ethyl acetate 2:1). $[\alpha]_{\mathrm{D}}=+33.8\left(\right.$ c 1.0, $\left.\mathrm{CHCl}_{3}\right) . \mathrm{NMR}$ data: $\delta_{\mathrm{H}}\left(\mathrm{CDCl}_{3}\right) 7.18(\mathrm{~m}, 4 \mathrm{H}$, $\left.2 \mathrm{Ar}+H \mathrm{C}=\mathrm{C} H \mathrm{NO}_{2}\right), 6.95(\mathrm{~m}, 3 \mathrm{H}, \mathrm{Ar}), 5.68\left(\mathrm{~m}, 1 \mathrm{H}, H \mathrm{C}=\mathrm{CH}_{2}\right), 5.36\left(\mathrm{dd}, \mathrm{H}-3,{ }^{3} J=4.63 \mathrm{~Hz}, J=\right.$ 
$1.7 \mathrm{~Hz}), 5.24(\mathrm{~m}, 2 \mathrm{H}, \mathrm{HC}=\mathrm{C} H H), 4.53\left(\mathrm{dd}, \mathrm{H}-4, J=6.6 \mathrm{~Hz}, J_{3,4}=4.6 \mathrm{~Hz}\right), 3.55(\mathrm{~m}, \mathrm{NCH} H)$, $3.21(\mathrm{~m}, \quad \mathrm{NCHH}), 2.37(\mathrm{~m}, 2 \mathrm{H} \mathrm{CHH}) . \quad \delta_{\mathrm{C}}\left(\mathrm{CDCl}_{3}\right) 165.0(\mathrm{NC}=\mathrm{O}), 157.0(\mathrm{C}), 142.8$ $\left(\mathrm{CH}=\mathrm{CHNO}_{2}\right), 135.3(\mathrm{CH}=\mathrm{CH}), 134.2(\mathrm{CH}=\mathrm{CH}), 129.8(\mathrm{CH}), 122.9(\mathrm{CH}), 118.1\left(\mathrm{CH}=\mathrm{CH}_{2}\right)$, $115.5(\mathrm{CH}), 82.4(\mathrm{C}-3), 55.9(\mathrm{C}-4), 40.3\left(\mathrm{~N}_{-} \mathrm{CH}_{2}\right), 32.0\left(\mathrm{CH}_{2}\right)$. IR $\left(\mathrm{CHCl}_{3}, \mathrm{~cm}^{-1}\right): v 1768,1598$. MS (EI), $m / z: 289\left(\mathrm{M}^{+}+1,100\right), 242$ (79), 195 (49), 150 (71).

Nitroalkene $( \pm)-7$ c. From $160 \mathrm{mg}(0.65 \mathrm{mmol})$ of aldehyde $( \pm)-1 \mathrm{e}, 136 \mathrm{mg}(72 \%)$ of compound $( \pm)-7 \mathrm{c}$ was obtained as a orange solid after purification by flash chromatography (hexanes/ethyl acetate 1:1). Mp: $119-121{ }^{\circ} \mathrm{C}$ (hexanes/ethyl acetate). NMR data: $\delta_{\mathrm{H}}\left(\mathrm{CDCl}_{3}\right) 7.25(\mathrm{dd}, J=13.5$, $\left.5.9 \mathrm{~Hz}, H \mathrm{C}=\mathrm{CHNO}_{2}\right), 7.18(\mathrm{~d}, 2 \mathrm{H}, J=8.9 \mathrm{~Hz}, \mathrm{Ar}), 6.97\left(\mathrm{~d}, J=13.5 \mathrm{~Hz}, \mathrm{HC}=\mathrm{C} H \mathrm{NO}_{2}\right), 6.80(\mathrm{~d}$, $2 \mathrm{H}, J=8.9 \mathrm{~Hz}, \mathrm{Ar}), 5.72\left(\mathrm{~m}, H \mathrm{C}=\mathrm{CH}_{2}\right), 5.10(\mathrm{~m}, 2 \mathrm{H}, \mathrm{CH}=\mathrm{CHH}), 4.76(\mathrm{t}, \mathrm{H}-4, J=5.9 \mathrm{~Hz}),$, $\left(\mathrm{m}, \mathrm{H}-3\right.$ and $\left.\mathrm{O}_{-} \mathrm{CH}_{3}\right), 2.60(\mathrm{~m}, \mathrm{CHH}), 2.25(\mathrm{~m}, \mathrm{CHH}) . \delta_{\mathrm{C}}\left(\mathrm{CDCl}_{3}\right) 165.8(\mathrm{NC}=\mathrm{O}), 157.6(\mathrm{C})$, 144.7, 136.4, 134.9, 129.8, $118.2(\mathrm{CH}), 117.0,114.7(\mathrm{CH}), 64.5,55.7\left(\mathrm{O}_{-} \mathrm{CH}_{3}\right)$, 53.4, 29.5. IR $\left(\mathrm{KBr}, \mathrm{cm}^{-1}\right): v$ 1751. MS (EI), $m / z: 292\left(\mathrm{M}^{+}+1,32\right), 149(100)$.

\section{General procedure for the synthesis of nitroalkanes 8}

To a solution of the corresponding nitroalkene $(1.0 \mathrm{mmol})$ in dichloromethane $(4.0 \mathrm{~mL})$ and methanol $(0.4 \mathrm{~mL})$ was added tributyl tin hydride $(0.31 \mathrm{mg}, 1.2 \mathrm{mmol})$, and the mixture was stirred at room temperature for $24 \mathrm{~h}$. Evaporation of the solvent under reduced pressure gave an oil, which was triturated with ethanol and filtered off to give the corresponding nitroalkane, which were purified by chromatography on silica gel.

Nitroalkane (+)-8a. From $180 \mathrm{mg}(0.66 \mathrm{mmol})$ of nitroalkene (+)-7a, $127 \mathrm{mg}(70 \%)$ of compound $(+)-8 a$ was obtained as a yellow oil after purification by flash chromatography (hexanes/ethyl acetate 2:1). $[\alpha]_{\mathrm{D}}=+135.1\left(c 0.8, \mathrm{CHCl}_{3}\right)$. NMR data: $\delta_{\mathrm{H}}\left(\mathrm{CDCl}_{3}\right) 7.30(\mathrm{~m}, 2 \mathrm{H}$, $\mathrm{Ar}), 7.05(\mathrm{~m}, 3 \mathrm{H}, \mathrm{Ar}), 5.80\left(\mathrm{~m}, \mathrm{HC}=\mathrm{CH}_{2}\right), 5.33(\mathrm{~m}, 2 \mathrm{H}, \mathrm{CH}=\mathrm{CHH}), 5.30\left(\mathrm{~d}, \mathrm{H}-3,{ }^{3} J=4.9 \mathrm{~Hz}\right)$, 4.52 (td, $2 \mathrm{H}, J=6.8 \mathrm{~Hz}, J=2.4 \mathrm{~Hz}, \mathrm{C} H H \mathrm{NO}_{2}$ ), 4.04 (ddt, $J=15.6 \mathrm{~Hz}, J=5.8 \mathrm{~Hz}, J=1.2 \mathrm{~Hz}$, $\mathrm{NCH} H), 4.07$ (m, H-4), 3.78 (ddt, $J=15.6 \mathrm{~Hz}, J=6.6 \mathrm{~Hz}, J=1.2 \mathrm{~Hz}, \mathrm{NCHH}), 2.50(\mathrm{~m}, 2 \mathrm{H}$, $\mathrm{CHH}) . \delta_{\mathrm{C}}\left(\mathrm{CDCl}_{3}\right) 165.5(\mathrm{NC}=\mathrm{O}), 157.3(\mathrm{C}), 131.1\left(\mathrm{CH}=\mathrm{CH}_{2}\right), 129.9(\mathrm{CH}), 122.9\left(\mathrm{CH}=\mathrm{CH}_{2}\right)$, $119.9(\mathrm{CH}), 115.8(\mathrm{CH}), 80.9(\mathrm{C}-3), 72.0\left(\mathrm{CH}_{2} \mathrm{NO}_{2}\right), 55.1(\mathrm{C}-4), 43.7\left(\mathrm{~N}-\mathrm{CH}_{2}\right), 26.4\left(\mathrm{CH}_{2}\right)$. IR $\left(\mathrm{CHCl}_{3}, \mathrm{~cm}^{-1}\right): v 1760,1598$. MS (EI), $m / z: 277\left(\mathrm{M}^{+}+1,100\right), 249$ (28).

Nitroalkane (+)-8b. From $120 \mathrm{mg}(0.42 \mathrm{mmol})$ of nitroalkene (+)-7b, $73 \mathrm{mg}(60 \%)$ of compound $(+)-8 \mathbf{b}$ was obtained as a yellow oil after purification by flash chromatography (hexanes/ethyl acetate 1:1). $[\alpha]_{\mathrm{D}}=+87.6\left(c 2.0, \mathrm{CHCl}_{3}\right)$. NMR data: $\delta_{\mathrm{H}}\left(\mathrm{CDCl}_{3}\right) 7.24(\mathrm{~m}, 2 \mathrm{H}$, Ar), $6.98(\mathrm{~m}, 3 \mathrm{H}, \mathrm{Ar}), 5.72\left(\mathrm{~m}, H \mathrm{C}=\mathrm{CH}_{2}\right), 5.22\left(\mathrm{~d}, \mathrm{H}-3,{ }^{3} \mathrm{~J}=4.9 \mathrm{~Hz}\right), 5.11(\mathrm{~m}, 2 \mathrm{H}, \mathrm{CH}=\mathrm{CHH})$, $4.44\left(\mathrm{~m}, 2 \mathrm{H}, \mathrm{CH} \mathrm{NO}_{2}\right), 4.04\left(\mathrm{dt}, \mathrm{H}-4, J=8.1 \mathrm{~Hz},{ }^{3} J=4.9 \mathrm{~Hz},\right), 3.58(\mathrm{~m}, \mathrm{NCH} H), 3.14(\mathrm{~m}$, $\mathrm{NCHH}), 2.36\left(\mathrm{~m}, 4 \mathrm{H}, \mathrm{CH}_{2}\right) . \delta_{\mathrm{C}}\left(\mathrm{CDCl}_{3}\right) 165.6(\mathrm{NC}=\mathrm{O}), 157.1(\mathrm{C}), 134.3\left(\mathrm{CH}=\mathrm{CH}_{2}\right), 129.7$ $(\mathrm{CH}), 122.7(\mathrm{CH}), 117.8\left(\mathrm{CH}=\mathrm{CH}_{2}\right), 115.6(\mathrm{CH}), 80.4(\mathrm{C} 3), 71.8\left(\mathrm{CH}_{2} \mathrm{NO}_{2}\right), 54.8(\mathrm{C} 4), 39.8$ $\left(\mathrm{CH}_{2}\right), 32.0\left(\mathrm{CH}_{2}\right), 28.1\left(\mathrm{CH}_{2}\right)$. IR $\left(\mathrm{CHCl}_{3}, \mathrm{~cm}^{-1}\right) \vee 1757 . \mathrm{MS}(\mathrm{EI}), \mathrm{m} / z: 291\left(\mathrm{M}^{+}+1,100\right), 263$ (17).

Nitroalkane ( \pm )-8c. From $60 \mathrm{mg}(0.21 \mathrm{mmol})$ of nitroalkene $( \pm)-7 \mathrm{c}, 49 \mathrm{mg}(81 \%)$ of compound $( \pm)-8 \mathrm{c}$ was obtained as a orange solid after purification by flash chromatography (hexanes/ethyl 
acetate $\left.2: 1,1 \% \mathrm{Et}_{3} \mathrm{~N}\right)$. Mp: $91-93{ }^{\circ} \mathrm{C}$ (hexanes/ethyl acetate). $\mathrm{NMR}$ data: $\delta_{\mathrm{H}}\left(\mathrm{CDCl}_{3}\right) 7.23(\mathrm{~m}$, $2 \mathrm{H}, \mathrm{Ar}), 6.77(\mathrm{~m}, 2 \mathrm{H}, \mathrm{Ar}), 5.85\left(\mathrm{~m}, \mathrm{HC}=\mathrm{CH}_{2}\right), 5.12(\mathrm{~m}, 2 \mathrm{H}, \mathrm{CH}=\mathrm{CHH}), 4.37(\mathrm{td}, 2 \mathrm{H}, J=6.2 \mathrm{~Hz}$, $\left.J=1.8 \mathrm{~Hz}, \mathrm{CHHNO}_{2}\right), 4.20\left(\mathrm{ddd}, \mathrm{H}-3, J=9.7 \mathrm{~Hz},{ }^{3} J=5.4 \mathrm{~Hz}, J=4.4 \mathrm{~Hz}\right), 3.08\left(\mathrm{~s}, 3 \mathrm{H}, \mathrm{O}-\mathrm{CH}_{3}\right)$, $3.48\left(\mathrm{dt}, \mathrm{H}-4, J=9.4 \mathrm{~Hz},{ }^{3} J=5.4 \mathrm{~Hz}\right), 2.62(\mathrm{~m}, 2 \mathrm{H}, \mathrm{CHH}), 2.29(\mathrm{~m}, 2 \mathrm{H}, \mathrm{CHH}) . \delta_{\mathrm{C}}\left(\mathrm{CDCl}_{3}\right)$ $165.8(\mathrm{NC}=\mathrm{O}), 156.5,134.8,129.8,119.5,117.1,114.6,72.2,55.4,51.9,50.8,28.5$, 25.6. IR $\left(\mathrm{KBr}, \mathrm{cm}^{-1}\right): v 1735 \mathrm{MS}(\mathrm{EI}), \mathrm{m} / z: 290\left(\mathrm{M}^{+}, 45\right), 291$ (7), 149 (100), 134 (59).

Procedure for the synthesis of tricycle (-)-9b. $13 \%$ Aqueous sodium hypochlorite $(0.6 \mathrm{mmol})$ was added, drop by drop, at $0^{\circ} \mathrm{C}$, to a well stirred solution of oxime $(+)-5 \mathbf{b}(50 \mathrm{mg}, 0.25 \mathrm{mmol})$ and triethylamine $(0.5 \%)$ in anhydrous dichloromethane $(1.5 \mathrm{~mL})$. The mixture was allowed to heat to room temperature and then was stirred during $26 \mathrm{~h}$. The mixture was diluted with $\mathrm{H}_{2} \mathrm{O}$ and was extract with dichloromethane. The extract organic was dried $\left(\mathrm{MgSO}_{4}\right)$ and concentrated under reduced pressure. Flash column chromatography of this mixture eluting with hexanes/ethyl acetate (1:2) gave $12 \mathrm{mg}(25 \%)$ of compound (-)-9b as a white solid. Mp: 127-128 ${ }^{\circ} \mathrm{C}$ (hexanes/ethyl acetate). NMR data: $\delta_{\mathrm{H}}\left(\mathrm{CDCl}_{3}\right) 4.84\left(\mathrm{dd}, \mathrm{H}-10,{ }^{3} \mathrm{~J}=4.1 \mathrm{~Hz},{ }^{5} \mathrm{~J}=0.9 \mathrm{~Hz}\right), 4.66$ (dd, H-5, $\left.{ }^{3} J=10.5 \mathrm{~Hz},{ }^{2} J=8.0 \mathrm{~Hz}\right) 4.42$ (dd, H-9, ${ }^{3} J=4.1 \mathrm{~Hz},{ }^{4} J=1.5 \mathrm{~Hz}$ ), 3.96 (ddd, H-2, ${ }^{2} J$ $\left.=14.1 \mathrm{~Hz},{ }^{3} \mathrm{~J}=4.9 \mathrm{~Hz},{ }^{3} \mathrm{~J}=1.7 \mathrm{~Hz}\right), 3.76\left(\mathrm{dd}, \mathrm{H}-5,{ }^{3} \mathrm{~J}=12.7 \mathrm{~Hz},{ }^{2} \mathrm{~J}=8.0 \mathrm{~Hz}\right), 3.62\left(\mathrm{~s}, \mathrm{O}-\mathrm{CH}_{3}\right)$, 3.41 (m, H-4), 2.80 (tdd, H-2, $J=12.7 \mathrm{~Hz},{ }^{3} J=3.3 \mathrm{~Hz},{ }^{5} J=0.9 \mathrm{~Hz}$ ), 2.05 (m, H-3'), 1.59 (qd, H$\left.3, J=12.6 \mathrm{~Hz},{ }^{3} J=4.9 \mathrm{~Hz}\right) . \delta_{\mathrm{C}}\left(\mathrm{CDCl}_{3}\right) 167.1(\mathrm{C}-11), 150.6(\mathrm{C}-8), 86.5(\mathrm{C}-10), 72.5(\mathrm{C}-5), 59.6$

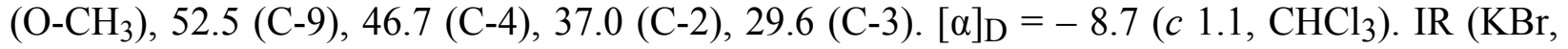
$\left.\mathrm{cm}^{-1}\right): v 1749$. MS (EI), m/z : $197\left(\mathrm{M}^{+}+1,15\right), 196\left(\mathrm{M}^{+}, 100\right), 140$ (29), 125 (67), 82 (71). Anal. Calcd. for $\mathrm{C}_{14} \mathrm{H}_{14} \mathrm{~N}_{2} \mathrm{O}_{3}$ (258.27): C, 65.11; H, 5.46; N, 10.85. Found: C, 65.21; H, 5.40; N, 10.80.

Procedure for the synthesis of tricycles (+)-9c and (+)-9d. To a solution of $N$ chlorosuccinimide $(0.36 \mathrm{mmol})$ in anhydrous dichloromethane $(2 \mathrm{~mL})$, under argon atmosphere, at $0^{\circ} \mathrm{C}$ was added dropwise a solution of oxime $(+)-5 \mathrm{c}(100 \mathrm{mg},(.36 \mathrm{mmol})$ in anhydrous dichloromethane $(4 \mathrm{~mL})$. The mixture was stirred for $5 \mathrm{~h}$ until reached room temperature. Then, triethylamine was added $(1 \mathrm{mmol})$ and the mixture was stirred for $12 \mathrm{~h}$. The organic mixture was washed with water, dried $\left(\mathrm{MgSO}_{4}\right)$ and concentrated under reduced pressure. Chromatography of this mixture eluting with hexanes/ether (1:1.5) allowed to obtain $23 \mathrm{mg}(25 \%)$ of compound (+)9c as a white solid, mp: $166-167{ }^{\circ} \mathrm{C}$ (hexanes/ethyl acetate), $[\alpha]_{\mathrm{D}}=+243.0\left(\mathrm{c} 1.0, \mathrm{CHCl}_{3}\right)$, as well as $9 \mathrm{mg}(10 \%)$ of compound (+)-9d as a white solid, mp 127-129 ${ }^{\circ} \mathrm{C}$ (hexanes/ethyl acetate), $[\alpha]_{\mathrm{D}}=+3.3\left(\right.$ c $\left.0.9, \mathrm{CHCl}_{3}\right)$. NMR data of tricycle $(+)-9 \mathrm{c}: \delta_{\mathrm{H}}\left(\mathrm{CDCl}_{3}\right) 7.56(\mathrm{~d}, 2 \mathrm{H}, J=$ $8.9 \mathrm{~Hz}, \mathrm{Ar}), 6.83(\mathrm{~d}, 2 \mathrm{H}, J=8.9 \mathrm{~Hz}, \mathrm{Ar}) 5.23\left(\mathrm{~d}, \mathrm{H}-10,{ }^{3} \mathrm{~J}=5.5 \mathrm{~Hz}\right), 4.94\left(\mathrm{dd}, \mathrm{H}-2,{ }^{3} J=5.5 \mathrm{~Hz}\right.$, $\left.{ }^{4} J=0.6 \mathrm{~Hz}\right), 4.56(\mathrm{dd}, J=9.8 \mathrm{~Hz}, J=8.1 \mathrm{~Hz}, \mathrm{OCH} H), 4.24(\mathrm{dd}, J=10.9 \mathrm{~Hz}, J=5.1 \mathrm{~Hz}$, $\mathrm{OCH} H), 3.72\left(\mathrm{~s}, \mathrm{O}-\mathrm{CH}_{3}\right), 3.69(\mathrm{~m}, 2 \mathrm{H}, \mathrm{OCHH}, \mathrm{OCHH}), 3.42(\mathrm{~m}, \mathrm{H}-7) . \delta_{\mathrm{C}}\left(\mathrm{CDCl}_{3}\right) 162.7(\mathrm{C}-$ 11), 156.9 (C), 154.1 (C-3), 129.9 (C), $119.3(\mathrm{CH}), 114.3(\mathrm{CH}), 77.8$ (C-10), 69.7 (O-CH $), 65.4$ $\left(\mathrm{O}-\mathrm{CH}_{2}\right), 55.5\left(\mathrm{O}-\mathrm{CH}_{3}\right), 48.2(\mathrm{C}-2), 44.9(\mathrm{C}-7)$. IR $\left(\mathrm{KBr}, \mathrm{cm}^{-1}\right): v 1755,1508$. Anal. Calcd. for $\mathrm{C}_{14} \mathrm{H}_{14} \mathrm{~N}_{2} \mathrm{O}_{4}$ (274.27): C, 61.31; H, 5.14; N, 10.21. Found : C, 61.23; H, 5.18; N, 10.17. 
NMR data of tricycle (+)-9d: $\delta_{\mathrm{H}}\left(\mathrm{CDCl}_{3}\right) 7.44(\mathrm{~d}, 2 \mathrm{H}, J=8.9 \mathrm{~Hz}, \mathrm{Ar}), 6.89(\mathrm{~d}, 2 \mathrm{H}, J=8.9$ $\mathrm{Hz}, \mathrm{Ar}), 5.32$ (d, H-10, $\left.{ }^{3} J=5.4 \mathrm{~Hz}\right), 5.05$ (d, H-2, $\left.{ }^{3} J=5.4 \mathrm{~Hz}\right), 4.82$ (dd, $J=10.7 \mathrm{~Hz}, J=8.3 \mathrm{~Hz}$, $\mathrm{OCH} H), 4.36(\mathrm{dd}, J=12.7 \mathrm{~Hz}, J=7.3 \mathrm{~Hz}, \mathrm{OCH} H), 4.06(\mathrm{dd}, J=13.7 \mathrm{~Hz}, J=8.3 \mathrm{~Hz}, \mathrm{OCHH})$, $3.93(\mathrm{dd}, J=12.7 \mathrm{~Hz}, J=1.9 \mathrm{~Hz}, \mathrm{OCHH}), 3.80\left(\mathrm{~s}, 3 \mathrm{H}, \mathrm{O}-\mathrm{CH}_{3}\right), 3.50(\mathrm{~m}, \mathrm{H}-7) . \delta_{\mathrm{C}}\left(\mathrm{CDCl}_{3}\right) 161.9$ (C-11), $157.1(\mathrm{C}), 155.2$ (C-3), $130.6(\mathrm{C}), 118.2(\mathrm{CH}), 114.7(\mathrm{CH}), 82.7(\mathrm{C}-10), 75.8\left(\mathrm{O}-\mathrm{CH}_{2}\right)$, $65.8\left(\mathrm{O}-\mathrm{CH}_{2}\right), 55.5\left(\mathrm{O}-\mathrm{CH}_{3}\right), 50.4(\mathrm{C}-2), 42.0(\mathrm{C}-7)$. Anal. Calcd. for $\mathrm{C}_{14} \mathrm{H}_{14} \mathrm{~N}_{2} \mathrm{O}_{4}$ (274.27): C, 61.31; H, 5.14; N, 10.21. Found: C, 61.40; H, 5.10; N, 10.16.

\section{General procedure for the synthesis of cycloadducts 10}

To a solution of the corresponding nitroalkane $8(1.0 \mathrm{mmol})$ in dry chloroform $(30 \mathrm{~mL})$, at room temperature, was added phenyl isocyanate $(2.3 \mathrm{mmol})$, followed by triethylamine $(0.3 \mathrm{mmol})$. The mixture was stirred for $10 \mathrm{~h}$, during which time diphenylurea precipitated, and was separated as a solid by filtration. The filtrate was washed with water and dried $\left(\mathrm{MgSO}_{4}\right)$. The solvent was removed under reduced pressure to leave a semisolid, which was purified by chromatograpy on silica gel.

Cycloadduct (+)-10a. From $50 \mathrm{mg}(0.18 \mathrm{mmol})$ of nitroalkane (+)-8a, $38 \mathrm{mg}(83 \%)$ of compound $(+)-10 a$ was obtained as a white solid after purification by flash chromatography (chloroform/ethyl acetate 5:1). Mp: $181-182{ }^{\circ} \mathrm{C}$ (chloroform /ethyl acetate). $[\alpha]_{\mathrm{D}}=+41.7$ (c 0.9, $\left.\mathrm{CHCl}_{3}\right)$. NMR data: $\delta_{\mathrm{H}}\left(\mathrm{CDCl}_{3}\right) 7.25$ (m, 2H, Ar), 6.90 (m, 3H, Ar), 5.37 (dd, H-10, J=4.1 Hz, $J=1.2 \mathrm{~Hz}$ ), 4.51 (dd, H-4', $\left.{ }^{3} J=10.7 \mathrm{~Hz},{ }^{2} J=8.5 \mathrm{~Hz}\right), 4.37$ (dd, H-2', $\left.{ }^{2} J=13.1,{ }^{3} J=7.8 \mathrm{~Hz}\right)$, 3.97 (m, H-9), 3.92 (t, H-4, $J=9.3 \mathrm{~Hz}) 3.42$ (m, H-3), 2.81 (dd, H-8', ${ }^{2} J=14.1 \mathrm{~Hz},{ }^{3} J=5.4 \mathrm{~Hz}$ ), $2.78(\mathrm{~m}, \mathrm{H}-2), 2.61\left(\mathrm{ddd}, \mathrm{H}-8,{ }^{2} J=14.1 \mathrm{~Hz},{ }^{3} J=10.2 \mathrm{~Hz},{ }^{4} J=1.5 \mathrm{~Hz}\right) . \delta_{\mathrm{C}}\left(\mathrm{CDCl}_{3}\right) 164.3(\mathrm{C} 11)$, $156.7(\mathrm{C}), 154.6$ (C-7), $129.7(\mathrm{CH}), 122.6(\mathrm{CH}), 115.0(\mathrm{CH}), 81.1$ (C-10), 71.2 (C-4), 53.9 (C-9), 46.3 (C-3), 43.4 (C-2), 23.8 (C-8). IR (KBr, cm-1): v 1751. MS (EI), $m / z: 258$ (M , 4), 165 (100), 77 (31). Anal. Calcd. for $\mathrm{C}_{14} \mathrm{H}_{14} \mathrm{~N}_{2} \mathrm{O}_{3}$ (258.27): C, 65.11; H, 5.46; N, 10.85. Found: C, 65.02; H, 5.40; N, 10.90.

Cycloadduct 10b. From $45 \mathrm{mg}(0.15 \mathrm{mmol})$ of nitroalkane $(+)-8 \mathbf{b}, 21 \mathrm{mg}(50 \%)$ of compound 10b, as a mixture $(63 / 37)$ of isomers, was obtained as a colorless oil after purification by flash chromatography (dichloromethane/ethyl acetate 9:1). NMR data: $\delta_{\mathrm{H}}\left(\mathrm{CDCl}_{3}\right) 1.90(\mathrm{~m}, 2 \mathrm{H}, \mathrm{H}-3$ M, H-3 m, H-3'M, H-3'), 2.54 (ddd, 0.63H, H-9, $J=14.2$ Hz, $J=11.7$ Hz, $J=1.1$ Hz), 2.95 (m, $2 \mathrm{H}), 3.33(\mathrm{~m}, 2 \mathrm{H}), 3.73(\mathrm{ddd}, 0.63 \mathrm{H}, J=11.4 \mathrm{~Hz}, J=4.2 \mathrm{~Hz}, J=2.6 \mathrm{~Hz}), 3.84$ (dd, 0.37H, H-5, $J=12.4 \mathrm{~Hz}, J=8.3 \mathrm{~Hz}), 4.02$ (t, 0.63H, H-5, $J=8.4 \mathrm{~Hz}), 4.11$ (m, 1.37H), 4.50 (dd, 0.63H, H5', $J=10.5 \mathrm{~Hz}, J=8.5 \mathrm{~Hz}), 4.54$ (dd, 0.37H, H-5', $J=8.3 \mathrm{~Hz}, J=7.3 \mathrm{~Hz}), 5.22$ (dd, 0.63H, H$11, J=4.2 \mathrm{~Hz}, J=1.1 \mathrm{~Hz}), 5.26(\mathrm{dd}, 0.37 \mathrm{H}, \mathrm{H}-11 J=4.6 \mathrm{~Hz}, J=1.1 \mathrm{~Hz}), 6.94(\mathrm{~m}, 3 \mathrm{H}, \mathrm{Ar}), 7.25$ $(\mathrm{m}, 2 \mathrm{H}, \mathrm{Ar}) . \delta_{\mathrm{C}}\left(\mathrm{CDCl}_{3}\right) 164.8(\mathrm{C}-12, M), 164.5(\mathrm{C}-12, m), 158.9(\mathrm{C}-8, M), 158.2(\mathrm{C}-8, m)$, $157.0(\mathrm{C}, M+m), 129.7(\mathrm{CH}, M+m), 122.5(\mathrm{CH}, M+m), 115.3(\mathrm{CH}, m), 115.2(\mathrm{CH}, M), 80.8$ $(\mathrm{C}-11, m), 79.4(\mathrm{C}-11, M), 74.7(\mathrm{C}-5, M), 74.0(\mathrm{C}-5, m), 56.3(M), 56.1(\mathrm{~m}), 50.8(\mathrm{~m}), 50.3(M)$, $40.9(m), 40.0(M), 28.9,28.8,28.7,27.9$. $).$ IR $\left(\mathrm{CHCl}_{3}, \mathrm{~cm}^{-1}\right): v 1750 . \mathrm{MS}(\mathrm{EI}), \mathrm{m} / z: 272\left(\mathrm{M}^{+}\right.$, 88), 271 (1), 149 (100), 123 (59). 
Cycloadducts $( \pm)$-10c and $( \pm)$-10d. From $100 \mathrm{mg}(0.35 \mathrm{mmol})$ of nitroalkane $( \pm)-8 \mathrm{c}$, and after flash chromatography eluting with ethyl acetate, $49 \mathrm{mg}(51 \%)$ of the compound $( \pm)-10 \mathrm{c}, \mathrm{mp} 143$ $144^{\circ} \mathrm{C}$ (white solid), and $11 \mathrm{mg}(11 \%)$ of compound $( \pm)-10 \mathrm{~d}, \mathrm{mp} 148-149^{\circ} \mathrm{C}$ (white solid), were obtained. NMR data of $( \pm)-10 \mathrm{c}: \delta_{\mathrm{H}}\left(\mathrm{CDCl}_{3}\right) 7.20(2 \mathrm{H}, J=9.0 \mathrm{~Hz}, \mathrm{Ar}), 6.80(2 \mathrm{H}, J=9.0 \mathrm{~Hz}, \mathrm{Ar})$, 4.49 (dd, H-7', $\left.{ }^{3} J=9.9 \mathrm{~Hz},{ }^{2} J=8.1 \mathrm{~Hz}\right), 4.42\left(\mathrm{td}, \mathrm{H} 2, J=5.4 \mathrm{~Hz},{ }^{3} J=1.1 \mathrm{~Hz}\right), 3.76\left(\mathrm{dd}, \mathrm{H}-7,{ }^{3} J\right.$ $\left.=11.1 \mathrm{~Hz},{ }^{2} J=8.1 \mathrm{~Hz}\right), 3.70\left(\mathrm{~s}, \mathrm{O}-\mathrm{CH}_{3}\right), 3.58\left(\mathrm{td}, \mathrm{H}-10, J=5.4 \mathrm{~Hz},{ }^{3} J=2.1 \mathrm{~Hz}\right), 3.44(\mathrm{~m}, \mathrm{H}-8)$, 3.22 (dd, H-3', $\left.{ }^{2} J=19.5 \mathrm{~Hz},{ }^{3} J=1.1 \mathrm{~Hz}\right), 2.83$ (ddd, H-3, ${ }^{2} J=19.5,{ }^{3} J=5.7 \mathrm{~Hz},{ }^{4} J=1.9 \mathrm{~Hz}$ ), 2.45 (ddd, H-9', $\left.{ }^{2} J=13.5 \mathrm{~Hz},{ }^{3} J=4.8 \mathrm{~Hz},{ }^{3} J=2.0 \mathrm{~Hz}\right), 1.65\left(\mathrm{td}, \mathrm{H}-9, J=13.5 \mathrm{~Hz},{ }^{3} J=5.7 \mathrm{~Hz}\right)$. $\delta_{\mathrm{C}}\left(\mathrm{CDCl}_{3}\right) 165.1(\mathrm{C}-11), 156.8(\mathrm{C} 4), 156.6(\mathrm{C}), 129.5(\mathrm{CH}), 118.6(\mathrm{CH}), 114.7(\mathrm{CH}), 72.7(\mathrm{C}-$

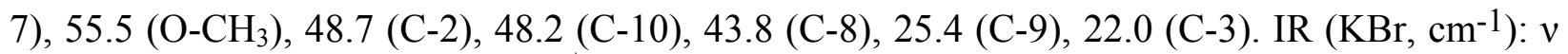
1747, 1515. MS (EI), m/z : $272\left(\mathrm{M}^{+}, 88\right), 273$ (18), 271 (5), 149 (100), 123 (59). IR (KBr, cm $\left.{ }^{-1}\right)$ : $v$ 1747, 1515. Anal. Calcd. for $\mathrm{C}_{15} \mathrm{H}_{16} \mathrm{~N}_{2} \mathrm{O}_{3}$ (272.30): C, 66.16; H, 5.92; N, 10.29. Found: C, 66.26; H, 5.89; N, 10.25. NMR data of $( \pm)-10 d: \delta_{\mathrm{H}}\left(\mathrm{CDCl}_{3}\right) 7.20$ (m, 2H, Ar), 6.80 (m, 2H, Ar), 4.56 (dd, H-7', $\left.{ }^{3} J=10.2 \mathrm{~Hz},{ }^{2} J=8.2 \mathrm{~Hz}\right), 4.48$ (ddd, H-2, ${ }^{3} J=5.3 \mathrm{~Hz},{ }^{3} J=4.5 \mathrm{~Hz},{ }^{3} J=2.2 \mathrm{~Hz}$ ), $3.85\left(\mathrm{dd}, \mathrm{H}-7,{ }^{3} \mathrm{~J}=14.1 \mathrm{~Hz},{ }^{2} \mathrm{~J}=8.2 \mathrm{~Hz}\right), 3.70\left(\mathrm{~s}, \mathrm{O}-\mathrm{CH}_{3}\right), 3.50$ (ddd, H-10, ${ }^{3} \mathrm{~J}=6.8 \mathrm{~Hz},{ }^{3} \mathrm{~J}=5.3$ $\left.\mathrm{Hz},{ }^{3} J=3.6 \mathrm{~Hz}\right), 3.36$ (m, H-8), 3.18 (dd, H-3, $\left.{ }^{2} J=15.0 \mathrm{~Hz},{ }^{3} J=2.2 \mathrm{~Hz}\right), 2.64$ (ddd, H-3, ${ }^{2} J=$ $\left.15.0,{ }^{3} J=4.5 \mathrm{~Hz},{ }^{4} J=1.5 \mathrm{~Hz}\right), 2.20\left(\mathrm{ddd}, \mathrm{H}-9,{ }^{2} J=15.5 \mathrm{~Hz},{ }^{3} J=8.8 \mathrm{~Hz},{ }^{3} J=6.8 \mathrm{~Hz}\right), 2.10(\mathrm{dt}$, $\left.\mathrm{H}-9,{ }^{2} J=15.5 \mathrm{~Hz}, J=3.6 \mathrm{~Hz}\right) . \delta_{\mathrm{C}}\left(\mathrm{CDCl}_{3}\right) 165.1(\mathrm{C}-11), 156.8(\mathrm{C} 4), 156.6(\mathrm{C}), 129.5(\mathrm{C}), 119.1$ $(\mathrm{CH}), 114.6(\mathrm{CH}), 74.8(\mathrm{C}-7), 55.5\left(\mathrm{O}-\mathrm{CH}_{3}\right), 51.9$ (C-2), 48.2 (C-10), 43.5 (C-8), 24.7 (C-9), 21.2 (C-3). IR (KBr, cm-1): v 1735, 1514. Anal. Calcd. for $\mathrm{C}_{15} \mathrm{H}_{16} \mathrm{~N}_{2} \mathrm{O}_{3}$ (272.30): C, 66.16; $\mathrm{H}$, 5.92; N, 10.29. Found: C, 66.08; H, 5.88; N, 10.33.

Procedure for the synthesis of tricycle 11. To a solution of nitroaldol 6a (200 $\mathrm{mg}, 0.68 \mathrm{mmol})$ and a catalytic amount of DMAP in dichloromethane at $0{ }^{\circ} \mathrm{C}$ under argon was slowly added triethylamine $(2.4 \mathrm{mmol})$ and acetic anhydride $(1.2 \mathrm{mmol})$. The mixture was stirred at room temperature for $17 \mathrm{~h}$, and then water was added. The solution was extract with ethyl acetate, the organic layer was dried $\left(\mathrm{MgSO}_{4}\right)$, and concentrated at reduced pressure. After chromatography on silica gel eluting with (hexanes/ethyl acetate 1:1) gave $53 \mathrm{mg}$ of compound $11(30 \%)$ as a mixture of isomers $(65 / 35)$. NMR data: $\delta_{\mathrm{H}}\left(\mathrm{CDCl}_{3}\right) 7.31(\mathrm{~m}, 4 \mathrm{H}, \mathrm{Ar}, \mathrm{M}, \mathrm{m}), 7.01(\mathrm{~m} 6 \mathrm{H}, \mathrm{Ar}, \mathrm{M}$, m), 5.95 (s, H-8, m), 5.92 (s, H-8, M), 5.73 (s, H-10, m), 5.66 (s, H-10, M), 4.66 (m, H-4'M, H4'm), 4.29 (m, H-2'M, H-2'm), 3.85 (m, H-3M, H-3m, H-4M, H-4m), 3.44 (m, H-2, m), 3.32 (m, $\mathrm{H}-2, \mathrm{M}) . \delta_{\mathrm{C}}\left(\mathrm{CDCl}_{3}\right) 163.1(\mathrm{C}-11, \mathrm{M}), 162.9(\mathrm{C}-11, \mathrm{~m}), 156.6$ (C, M, m), 152.4 (C-7, M), 152.1 (C-7, m), 146.7 (C-9, M), 146.4 (C-9, m), 129.7 (CH, M, m), 123.1 (C, M, m), 115.8 (CH, M), 115.6 (CH, m), 89.1 (C-8, M), 88.9 (C-8, m), 82.5 (C-10, M), 82.0 (C-10, m), 71.7 (C-4, M, m), 44.6 (C-3, M, m), 42.3 (C-2, m), 41.7 (C-2, M). ). IR $\left(\mathrm{CHCl}_{3}, \mathrm{~cm}^{-1}\right): v$ 1750. Anal. Calcd. for $\mathrm{C}_{14} \mathrm{H}_{12} \mathrm{~N}_{2} \mathrm{O}_{3}$ (256.26): C, 65.62; H, 4.72; N, 10. 39. Found: C, 65.73; H, 4.69; N, 10.44. 


\section{Acknowledgements}

Support for this work by the DGI-MCYT (Project BQU2000-0645) is gratefully acknowledged. One of us (E. S.) also thanks the DGES (MEC, Spain) for a predoctoral grant.

\section{References}

1. See, for example: (a) Setti, E. L.; Micetich, R. G. Curr. Med. Chem. 1998, 5, 101. (b) Georg, G. I. Ed. The Organic Chemistry of $\beta$-LactamsVCH: New York, 1993. (c) Neuhaus, F. C.; Georgeopapadakou, N. H. In Emerging Targets in Antibacterial and Antifungal Chemoterapy; Sutcliffe, J.; Georgeopapadakou, N. H., Eds.; Chapman and Hall: New York, 1992. (d) Page, M. I. Ed., The Chemistry of $\beta$-Lactams; Chapman and Hall: London, 1992.

2. For selected references, see: (a) Kanno, O.; Kawamoto, I. Tetrahedron 2000, 56, 5639. (b) Hanessian, S.; Reddy, B. Tetrahedron 1999, 55, 3427. (c) Biondi, S.; Pecunioso, A.; Busi, F.; Contini, S. A.; Donati, D.; Maffeis, M.; Pizzi, D. A.; Rossi, L.; Rossi, T.; Sabbatine, F. M. Tetrahedron 2000, 56, 5649. (d) Ghiron, C.; Rossi, T. The Chemistry of Trinems in Targets in Heterocyclic Systems-Chemistry and Properties, Attanasi, O. A.; Spinelli, D., Eds.; Societa Chimica Italiana: Rome, 1997; Vol. 1, pp 161-186. (e) Ngo, J.; Castañer, J. Drugs of the Future 1996, 21, 1238.

3. (a) Page, M. I.; Laws, A. P. Tetrahedron 2000, 56, 5631. (b) Bonneau, P. R.; Hasani, F.; Plouffe, C.; Malenfant, E.; LaPlante, S. R.; Guse, I.; Ogilvie, W. W.; Plante, R.; Davidson, W. C.; Hopkins, J. L.; Morelock, M. M.; Cordingley, M. G.; Deziel, R. J. Am. Chem. Soc. 1999, 121, 2965. (c) Vaccaro, W. D.; Davis Jr., H. R. Bioorg. Med. Chem. Lett. 1998, 8, 313. (d) Borthwick, A. D.; Weingarte, G.; Haley, T. M.; Tomaszewski, M.; Wang, W.; Hu, Z.; Bedard, J.; Jin, H.; Yuen, L.; Mansour, T. S. Bioorg. Med. Chem. Lett. 1998, 8, 365. (e) Han, W. T.; Trehan, A. K.; Wright, J. J. K.; Federici, M. E.; Seiler, S. M.; Meanwell, N. A. Bioorg. Med. Chem. 1995, 3, 1123.

4. For reviews, see: (a) Alcaide, B.; Almendros, P. Chem. Soc. Rev. 2001, 30, 226. (b) Alcaide, B.; Almendros, P. Org. Prep. Proced. Int. 2001, 33, 315. (c) Alcaide, B.; Almendros, P. Synlett 2002, 381. (d) Palomo, C.; Aizpurua, J. M.; Ganboa, I.; Oiarbide, M. Synlett 2001, 1813. (e) Palomo, C.; Aizpurua, J. M.; Ganboa, I.; Oiarbide, M. Amino-acids 1999, 16, 321. (f) Ojima, I.; Delaloge, F. Chem. Soc. Rev. 1997, 26, 377. (g) Ojima, I. Adv. Asym. Synth. 1995, 1, 95. (h) Manhas, M. S.; Wagle, D. R.; Chiang, J.; Bose, A. K. Heterocycles 1988, $27,1755$.

5. For a recent review on bi- and tricyclic- $\beta$-lactams with non-classical structure, see: Alcaide, B.; Almendros, P. Curr. Org. Chem. 2002, 6, 245.

6. (a) Padwa, A.; Pearson, W. H. In Synthetic Applications of 1,3-Dipolar Cycloaddition Chemistry; Wiley: Hoboken, 2003. (b) Gothelf, K. V. In Cycloaddition Reactions in Organic Synthesis; Kobayashi, S.; Jørgensen, K. A. Eds.; Wiley-VCH: Weinheim, 2002; 
Chapter 3, pp 211-247. (c) Karlsson, S.; Högberg, H.-E. Org. Prep. Proc. Int. 2001, 33, 103. (d) Gothelf, K. V.; Jørgensen, K. A. Chem. Commun. 2000, 1449. (e) Gothelf, K. V.; Jørgensen, K. A. Chem. Rev. 1998, 98, 863. (f) Padwa, A. In 1,3-Dipolar Cycloaddition Chemistry; Ed.; Wiley: New York, 1984; Vol. 1 and 2. (g) Torssell, K. B. G. In Nitrile Oxides, Nitrones and Nitronates in Organic Synthesis, VCH: Weinheim, 1998.

7. Sandanayaka, V. P.; Yang, Y. Org. Lett. 2000, 2, 3087.

8. (a) Hassner, A.; Keshava Murthy, K. S. Tetrahedron Lett. 1987, 28, 97. (b) Hassner, A.; Keshava Murthy, K. S.; Padwa, A.; Bullock, W. H.; Stull, P. D. J. Org. Chem. 1988, 53, 5063.

9. See, for instance: (a) Alcaide, B.; Almendros, P.; Alonso, J. M.; Aly, M. F. Chem. Eur. J. 2003, 9, 3415. (b) Alcaide, B.; Almendros, P.; Pardo, C.; Rodríguez-Ranera, C.; RodríguezVicente, A. J. Org. Chem. 2003, 68, 3106. (c) Alcaide, B.; Almendros, P.; Alonso, J. M.; Redondo, M. C. J. Org. Chem. 2003, 68, 1426. (d) Alcaide, B.; Almendros, P.; Aragoncillo, C. Chem. Eur. J. 2002, 8, 1719. (e) Alcaide, B.; Almendros, P.; Aragoncillo, C.; Redondo, M. C. Chem. Commun. 2002, 1472. (f) Alcaide, B.; Almendros, P.; Aragoncillo, C. Chem. Eur. J. 2002, 8, 3646.

10. (a) Alcaide, B.; Almendros, P.; Alonso, J. M.; Aly, M. F.; Pardo, C.; Sáez, E.; Torres, M. R. J. Org. Chem. 2002, 67, 7004. (b) Alcaide, B.; Sáez, E. Tetrahedron Lett. 2000, 41, 1647. (d) Alcaide, B; Alonso, J. M.; Aly, M. F.; Sáez, E.; Martínez-Alcázar, M. P.; HernándezCano, F. Tetrahedron Lett. 1999, 40, 5391.

11. Alcaide, B.; Pardo, C.; Sáez, E. Synlett 2002, 85.

12. (a) Alcaide, B.; Martín-Cantalejo, Y.; Plumet, J.; Rodríguez-López, J.; Sierra, M. A. Tetrahedron Lett. 1991, 32, 803. (b) Alcaide, B.; Martín-Cantalejo, Y.; Pérez-Castells, J.; Rodríguez-López, J.; Sierra, M. A.; Monge, A.; Pérez-García, V. J. Org. Chem. 1992, 57, 5921.

13. For recent reviews on the ketene-imine approach to $\beta$-lactams, see: (a) Palomo, C.; Aizpurua, J. M.; Ganboa, I.; Oiarbide, M. Eur. J. Org. Chem. 1999, 3223. (b) Tidwell, T. T. Ketenes; Wiley: New York, 1995, p 518. (c) Georg, G. I.; Ravikumar, V. T. In The Organic Chemistry of $\beta$-lactams; Georg, G. I., Ed.; VCH: Weinheim, New York, 1993, Chapter 3, p 295. (d) Van der Steen, F. H.; Van Koten, G. Tetrahedron 1991, 47, 7503. (e) Ghosez, L.; Marchand-Brynaert, J. In Comprehensive Organic Synthesis; Trost, B.; Fleming, I.; Eds. Pergamon: Oxford, 1991, Vol. 5, p 85.

14. Palomo, C.; Aizpurua, J. M.; Cossío, F. P.; García, J. M.; López, M. C.; Oiarbide, M. J. Org. Chem. 1990, 55, 2070.

15. Mukaiyama, T.; Hoshino, T. J. Am. Chem Soc. 1960, 82, 5339. 